Tohoku J. Exp. Med., 2003, 199, 127-134

\title{
Comparative Effect of Intra-Articular Administration of Hyaluronan and/or Cortisone with Evaluation of Malondialdehyde on Degenerative Osteoarthritis of the Rabbit's Knee
}

\author{
Gunhan Karakurum, Metin Karakok, ${ }^{1}$ Mehmet Tarakcioglu, ${ }^{2}$ N. Emrah \\ Kocer, ${ }^{1}$ Ramazan Kocabas ${ }^{2}$ and CAhit BagCi ${ }^{3}$ \\ Department of Orthopedics and Traumatology, ${ }^{1}$ Department of Pathology, \\ ${ }^{2}$ Department of Biochemistry, ${ }^{3}$ Department of Physiology, Gaziantep University \\ School of Medicine, Gaziantep, Turkey
}

Karakurum, G., Karakok, M, Tarakcioglu, M., Kocer, N.E., Kocabas, R and BAGCI, C. Comparative Effect of Intra-Articular Administration of Hyaluronan and/or Cortisone with Evaluation of Malondialdehyde on Degenerative Osteoarthritis of the Rabbit's Knee. Tohoku J. Exp. Med., 2003, 199(3), 127-134Hyaluronan and cortisone have controversial and an important role in the healing of degenerative osteoarthritis. The purpose of the research was to compare individual and combined effects of hyaluronan and cortisone on the healing of degenerative osteoarthritis and to determine the serum malondialdehyde level as a lipid peroxidation marker. A rabbit model was used in which a degenerative osteoarthritis was created in the articular cartilage by resection of anterior cruciate ligament. The rabbits divided into three groups namely were injected with hyaluronan (group A) and cortisone (group B) at days 31, 38 and 45. Cortisone at day 31 and hyaluronan at days 38 and 45 were injected to the third group (group C). We obtained blood samples from each rabbit to determine the malondialdehyde levels at days 1, 30, and 52. At day 52, 21 rabbits were sacrificed. In biopsies obtained from treated and untreated knees articular cartilage degeneration was examined by light microscopy. Histopathologically the healing rate was significantly higher in group $\mathrm{C}$ than the other groups. Degeneration decreased $72 \%$ in group A, $52 \%$ in group B and $88 \%$ in group C at day 22 . Malondialdehyde levels were $2.05 \pm 0.37$ in the control group, $1.94 \pm 0.54$ in group $\mathrm{A}, 1.98 \pm 0.37$ in group $\mathrm{B}$ and $1.55 \pm 0.41$ in goup $\mathrm{C}$. The malondialdehyde levels of group $\mathrm{A}$ and $\mathrm{B}$ were less than the control group (statistically insignificant, $p>0.05$ ). But, there were statistically significant values between control group and group $\mathrm{C}(\phi<0.05)$. The results showed that the combination of cortisone and hyaluronan is the most effective in the treatment of cartilage degeneration in the course of the ostearthritis and the malondialdehyde levels are correlated with the severity of degeneration. - hyaluronan; cortisone; malondialdehyde; histopathology; osteoar-

Received June 5, 2002; revision accepted for publication March 10, 2003.

Address for reprints: Metin Karakok, Inonu Cad. No. 35, 27100, Gaziantep, Turkey.

e-mail: metinkarakok@yahoo.com 
thritis

(C) 2003 Tohoku University Medical Press

Osteoarthritis is a relatively noninflammatory disorder of synovial joints. Joint cartilage is a dynamic tissue that reacts to acute or chronic trauma, infection or corticosteroid therapy by attempting to repair its matrix. This reaction results in the release of cartilage macromolecules into the body fluids. Analysis of these fluids has identified a limited number of somewhat tissue specific markers of altered cartilage metabolism. Analysis of serum is less specific and sensitive than analysis of the synovial fluid, but their use as research tools in clinical studies and experimental work in animal models is increasing (Wollheim 1999). Hyaluronan binds to the proteoglycan surface components of articular cartilage and provides an important boundary layer of lubrication (Nizolek and White 1981). The model we used involve joint immobilization and resection of the anterior cruciate ligament, as the means for reliably inducing joint degeneration (Yoshimi et al. 1994). After destroying cartilage, fragments of hyaluronan become free (Bora and Miller 1987). And this event triggers regeneration mechanism in degenerative arthritis (Carrabba et al. 1992). The production of collagen, protein and glycosaminoglicans are associated with proliferation and hypermetabolism (Bora and Miller 1987; Morales and Hascall 1989). That is, hyaluronan acts as a biologic stimulator in regenerative events, and somewhat in this fashion it helps reformation of the extracellular matrix (Bayliss et al. 1984). Administration of exogenous hyaluronan, probably increases this effect in both experimental animal and human osteoarthritis (Graf et al. 1938; Toyoshima 1978). In many experimental studies it was observed that the high molecular weight of $\mathrm{H}$ was more effective than the low molecular counterpart in the treatment of osteoarthritis (Philips 1989; Gotoh et al. 1993; Sakakibara et al. 1994).

Injection of cortisone may be very effective on osteoarthritis of the knee, but may cause severe side effects at the injected knees. For instance, when it is used in high and repeated doses, it leads to disappearance of chondrocytes on the cartilage surface, which in turn, leads to joint cartilage degeneration (Selami 1993).

Oxygen-derived free radicals appear to be involved in the pathogenesis of arthritic disorders. The production of oxygen derived free radicals leads to lipid peroxidation that has destructive effect on the cells (Alpaslan et al. 2000).

In this study, osteoarthritis was created in the right knees of 24 rabbits, to determine the combined and individual effects of hyaluronan and cortisone and the correlation between free oxygen radicals and osteoarthritis severity on the healing of degeneration process histopathologically.

\section{Materials AND METHODS}

This experimental study of 52 days' duration was performed in 24 rabbits. At first, the blood samples obtained from each rabbit were evaluated for malondialdehyde levels. While the rabbits were under endotracheal general anesthesia, their right knees were shaved off and sterilized properly. A one-centimeter long skin incision paralleling the patellar tendon was performed, the articular capsule was incised longitudinally and the anterior cruciate ligaments of the right knees of 24 rabbits were resected in order to induce degenerative osteoarthritis (Yoshimi et al. 1994). Then the wound was closed as usual. Clinical findings such as effusion in the knees and limping were followed throughout the study.

At day 30, malondialdehyde serum levels of 24 rabbits were evaluated before the operation. 
Tissue samples were obtained from the right knees of the three rabbits that were sacrificed at day 30. Tissue samples were fixed in $10 \%$ formaline solution, after the tissue process they were embedded in parafin blocks, four micron thick sections were taken and stained with Haemotoxylin-Eosin. These samples were examined histopathologically.

At days 31,38 and $45,0.3 \mathrm{ml} /$ day hyaluronan (Orthovisc, Biomeks Co., Woburn, MA, USA) was given (Tosun et al. 1997) into the right knees of 7 rabbits (group A) out of 21, and $15 \mathrm{mg} /$ day 6-metyl prednisonolone was injected into the knees of the 7 rabbits (group B) of the remaining 14 . In addition to the same single cortisone dose which was administered intraarticularly at day $31,0.3 \mathrm{ml} /$ day hyaluronan was given into the knees of the remaining 7 rabbits (group C) at days 38 and 45 .

At day 52 , malondialdehyde serum levels of each group were evaluated and all the rabbits were sacrificed. Blood samples were obtained from ear vessels and sera were removed by centrifugation at $4000 \mathrm{rpm}$ for 5 minutes.

Serum malondialdeyde (MDA) levels (as a biochemical marker for lipid peroxidation) were measured by a spectrophotometric method. MDA generation was evaluated by the assay of thiobarbituric acid (TBA)-reacting compounds. In particular, the addition of a solution of 0.2 $\mathrm{mL}$ of sodium dodecyl sulphate (SDS; 8.1\%), 1.5 $\mathrm{mL}$ of $20 \%$ acetic acid solution $(\mathrm{pH}=3.5), 1.5$ $\mathrm{mL}$ of $0.8 \%$ TBA $(\mathrm{pH}=3.5)$, and $0.6 \mathrm{~mL}$ of distilled water produced a chromogenic product which was extracted in n-butanol and pyridine. The organic layer was removed by centrifugation at $4000 \mathrm{rpm}$ for 12 minutes at $23^{\circ} \mathrm{C}$ and MDA was read at $532 \mathrm{~nm}$ and expressed as $\mathrm{nmol} / \mathrm{mL}$. The amount of TBARs was calculated as MDA equivalents using 1,1,3,3tetramethoxypropane as standard (Ohkawa et al. 1979).

Articular surfaces were divided to eight equal regions (4 regions for tibial, 4 regions for femoral) for histologically determining the severity of degeneration in the cartilage zones. Degeneration severity was rated as follows: absence of degeneration was considered negative (-), cartilage splitting in zone I (Marion and Sanford 1992) (Fig. 1) was mild degeneration or (1p), cartilage splitting in intermediate zone II (Marion and Sanford 1992) was moderate degeneration or (2p) (Fig. 2); cartilage splitting involving zone III (Marion and Sanford 1992) was considered as severe degeneration or (3p), and complete loss of cartilage layer was

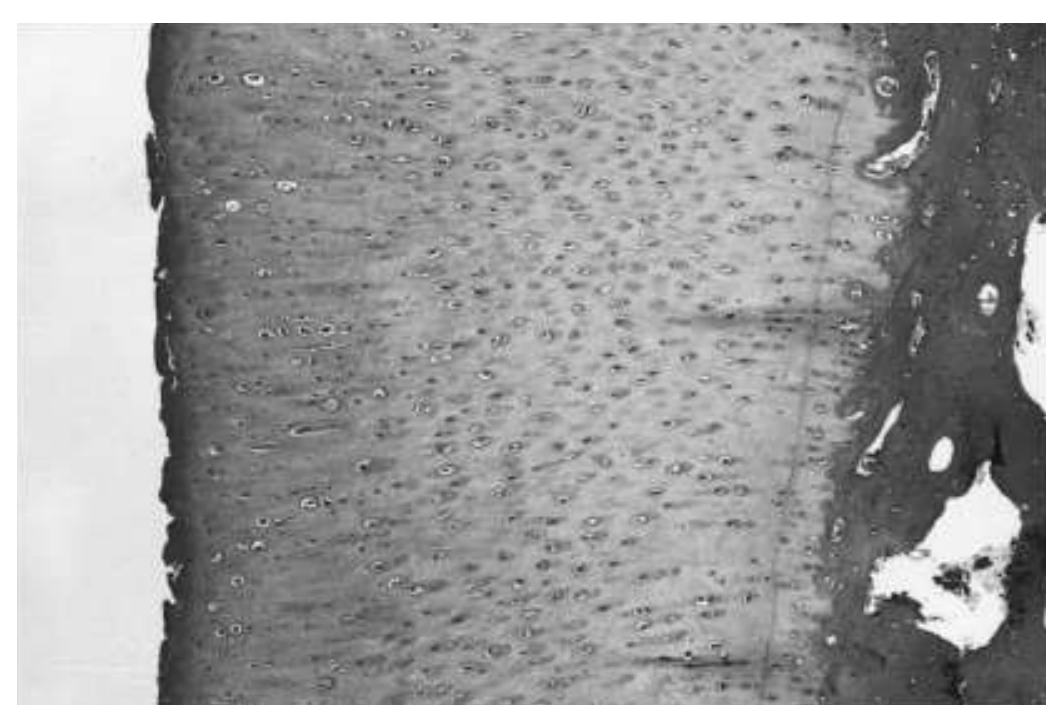

Fig. 1. Note the joint cartilage splitting till the zone-I which is described as a mild degeneration or (1p) $(\mathrm{H} \& \mathrm{E}, \times 100)$. 


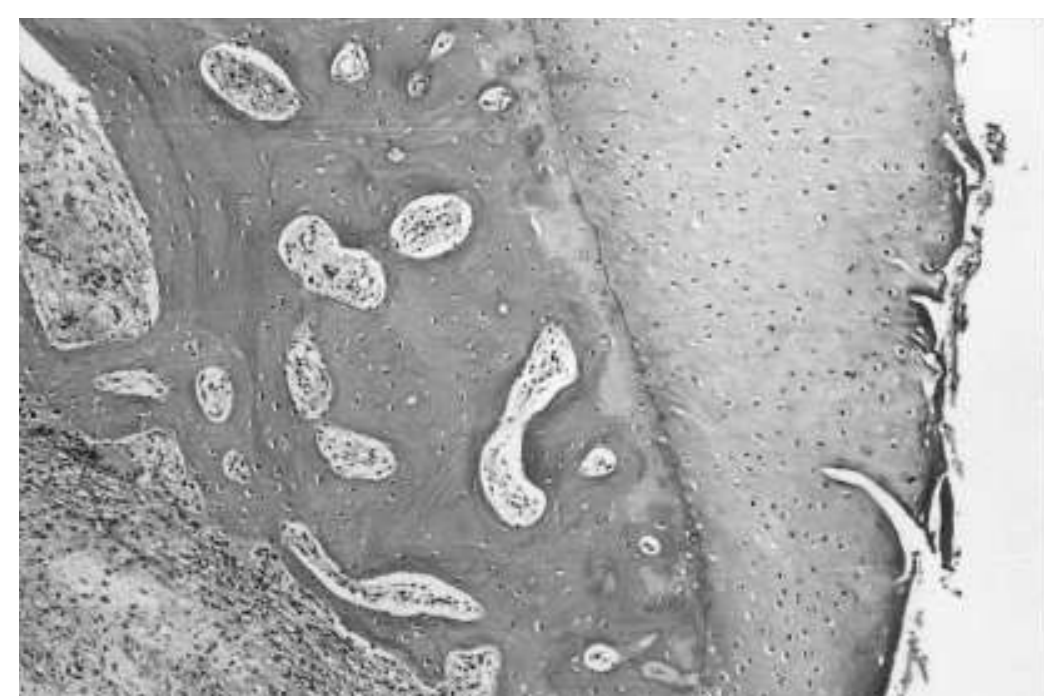

Fig. 2. A moderate degeneration or (2p) in a joint cartilage of a rabbit. Cartilage splitting reaches the intermediate zone $(\mathrm{H} \& \mathrm{E}, \times 100)$.

profound or (4p) degeneration. Degeneration severity observed in each location in each group was summed up to get the degeneration score. At 8 localizations, the degeneration was considered as mild, moderate and severe when the degeneration score was 1-24 (p), 25-48 (p) and 49-72 (p), respectively (Karakok et al. 2001).

The extension of degeneration in the groups was described as follows; degeneration more than one localization on the opposing surface or more than two localizations on nonopposing surfaces was considered diffuse or at most two localizations on the non-opposing or at most one localization on the opposing surfaces of the articular cartilage was considered local.

\section{Statistical analysis}

The results were expressed as mean \pm S.D. and were analyzed statistically by using oneway ANOVA and Tukey tests. The values of $p<0.05$ were considered significant for malondialdehyde.

\section{RESULTS}

A diffuse and moderate (25p) degeneration was observed in the right knees of the three rabbits that were sacrificed at day 30 and had been more intense in the joint regions exposed to pressure. We did not observe severe degeneration. Effusion disappeared after the day 10 of treatment, but limping was seen in several rabbits. Degeneration became focal and mild, (7p) in group $A,(12 p)$ in group B and (3p) in group $\mathrm{C}$ at day 52 (the 22 nd day of the treatment) regressing by $72 \%, 52 \%$ and $88 \%$ respectively as compared with the findings at day 30 . Under the light of these examinations, at day 52 , it was seen that the parameters were the worst in group $\mathrm{B}$ and the best in combined therapy (Table 1).

Malondialdehyde levels were $1.12 \pm 0.33$ (Mean \pm S.D) $\mathrm{nmol} / \mathrm{mL}$ in normal rabbits, 2.05 \pm $0.37 \mathrm{nmol} / \mathrm{mL}$ in degenerated (control group), $1.94 \pm 0.54 \mathrm{nmol} / \mathrm{mL}$ in group A, $1.98 \pm 0.37$ $\mathrm{nmol} / \mathrm{mL}$ in group B and $1.55 \pm 0.41 \mathrm{nmol} / \mathrm{mL}$ in goup C. The malondialdehyde levels of group $\mathrm{A}$ and $\mathrm{B}$ were less than control group, but these values were not statistically significant $(p>0$. $05)$. On the other hand, there were statistically significant values between control group and group C $(p<0.05)$ (Table 1$)$.

\section{DISCUSSION}

It was shown that hyaluronan had two steps of effect in animal experimental studies. 
TABLE 1. The histopathologic findings and malondialdehyde levels seen in the study

\begin{tabular}{ccccccc}
\hline & \multicolumn{3}{c}{ Histopathologic Values } & & \multicolumn{2}{c}{$\begin{array}{c}\text { Malondialdehyde Values } \\
\text { (nmol/mL) }\end{array}$} \\
\cline { 2 - 3 } \cline { 6 - 7 } & DS & MV & S.D. & & MV & S.D. \\
\hline In normal rabbit & 0 & 0 & 0 & & 1.12 & 0.33 \\
At day 30 & $25(\mathrm{p})$ & 3.13 & 0.99 & & 2.05 & 0.37 \\
At day 52 & & & & & \\
Group A & $7(\mathrm{p}) 72 \%^{\mathrm{c}}$ & 0.88 & 0.83 & & 1.94 & 0.55 \\
Group B & $12(\mathrm{p}) 52 \%^{\mathrm{c}}$ & 1.63 & 1.60 & & 1.97 & 0.37 \\
Group C & $3(\mathrm{p}) 88 \%^{\mathrm{c}}$ & 0.38 & 0.52 & & 1.55 & 0.41 \\
\hline
\end{tabular}

${ }^{a}$ The day after 30 days of the resection of anterior crucial ligaments.

b22nd day of the treatment.

'The healing rate as compared to day 30 .

DS, degeneration score; MV, mean value; s.D., standard deviation.

In the early stage after the injection it has lubrication effect, and in the late stage it prevents cartilage destruction and facilitates cartilage rearrangements (Ghosh et al. 1993).

Cortisone is known to have a strong antiinflamattory effect. Pietrogrande et al. (1991) claimed that painful symptoms and morning stiffness as well as the amount of the effusion ceased substantially within 7-14 days after cortisone therapy. Leardini et al. (1991), who studied 40 patients, reported that pain reduction and improvement in the joint function appeared immediately after the initial dose of cortisone.

According to Corrado et al. (1995) and Frizziero et al. (1988), a decrease in the effusion, synovial inflammation and edema occurred in humans and animals within five and two weeks, respectively.

There is frequently no correlation between the severity of pain and the degree of degenerative changes (Asheim and Linbald 1976; Nizolek and White 1981)

Jones and his colleagues administered either hyaluronan or cortisone in 63 patients once a week for 5 weeks. They evaluated the parameters of morning stiffness, pain, reduction in the joint mobility, joint effusion and increase in the local heat in these patients. As a result, they observed that initially there was not significant difference between the two treat- ments, except that the pain was less severe in hyaluronan group than cortisone group after a follow up of 6 moths, and the other parameters were similar (Jones et al. 1995).

In this study, effusion ceased not only in group B, but also in the other groups within ten days. Limping was observed in some of the rabbits from day 30 to the last day of the study.

In the study by Namiki et al. (1982), they graded his results as excellent in $26.7 \%$, good in $31.1 \%$, fair in $13.3 \%$ and poor in $28.9 \%$ in the treated knees. They noted an improvement immediately upon receiving an injection of hyaluronan in some patients, whereas in others, repeated hyaluronan administration was necessary to alleviate the arthritic conditions. Therefore, they suggested that the long-term, intraarthicular administration of hyaluronan might bring about satisfactory results in the treatment of patients with osteoarthritis.

In the present study, the result obtained from the group $\mathrm{C}$ was excellent and the healing rate was $88 \%$. Since this study lasted 52 days, it was not possible to evaluate the efficiency of hyaluronan in the longer term.

Pietrogrande et al. (1991) in an attempt to compare the activity and tolerability of $\mathrm{H}$ and cortisone, administered these drugs intraarthicularly to the patients who suffered from osteoarthritis in the knee, and for this purpose 
he administered hyaluronan once a week for five weeks and cortisone once a week for three weeks. They observed that steroid had a more rapid action, and there were significant differences between the two treatment options.

We claimed previously that better therapeutic effect of hyaluronan and the therapeutic efficiency of cortisone in the acute phase of degenerative joint disease due to septic arthritis, may be true for other type of degenerative arthritis as well (Karakok et al. 2001). For this, we administered the cortisone in the acute phase and then the hyalgan in traumatic osteoarthritis.

In this study, it was observed that the combination of hyaluronan and cortisone treatment was more effective than cortisone or hyaluronan alone.

Since the regression of arthritic lesions in the animals shows similarity to that of humans, many animal experimental studies have been performed (Peyron and Balazs 1974; Namiki et al. 1982).

This study showed that cartilage degeneration starts after resection of anterior cruciate ligaments, is more severe in the weight carrying localization, and also hyaluronan may prevent side effects of cortisone and there may be a synergic effect with cortisone. These results are compatible with the literature.

Pietrogrande and his colleagues (1991) reported that the high molecular weight hyaluronan not only improves the reological charateristics of synovial fluid, but also can control some stages of the inflammatory process, acting as a free radicals scavenger.

Kvam et al. (1995) claimed that high molecular weight hyaluronan shows a significant protective activity for chondrocytes against oxygen derived free radicals action, low molecular weight hyaluronan does not.

In a study by Cortivo et al. (1996), they reported that both hyaluronan and cortisone were capable, to some extent, of protecting tissues and cells from damage by reactive oxygen species; alpha-methyl prednisolone demon- strated the greatest protective effect.

Malondialdehyde has been identified as the product of lipid peroxidation that reacts with thiobarbituric acid to give a red species absorbing at $535 \mathrm{~nm}$ (Ustun et al. 2001). In this study, it provided objective corroboration for the severity of degeneration.

We conclude that the combination of hyaluronan and cortisone has a better therapeutic efficieny over hyaluronan and cortisone alone in the traumatic ostearthritis, evidenced by the biochemical parameters. Also, malondialdehyde levels may be a good indicator of degeneration degree in osteoarthritis.

\section{References}

Alpaslan, C., Bilgihan, A., Alpaslan, G.H., Guner, B., Ozgur, Yis, M. \& Erbas, D. (2000) Effect of arthrocentesis and sodium hyaluronate injection on nitrite, nitrate, and thiobarbituric acid-reactive substance levels in the synovial fluid. Oral Surg. Oral Med. Oral Pathol. Oral Radiol. Endod., 89, 689-690.

Asheim, A. \& Linbald, G. (1976) Intra-articular treatment of osteoarthritis in race horses with sodium hyaluronate. Acta Vet. Scand., 17, 379-394.

Bayliss, M.T., Ridgeway, G.D. \& Ali, S.Y. (1984) Delayed aggregation of proteoglycans in adult human articular cartilage. Biosci. Rep., 4, 827.

Bora, F.W. \& Miller, G. (1987) Joint physiology, cartilage metabolism and the etiology of osteoarthritis. Hand. Clin., 3, 325-326.

Carrabba, M., Paresce, E., Angelini, M., Zamboni, A.M., Bragantini, A., Paissan, A., Monilaroli, F. \& Parbellini, A. (1992) The intra-articular treatment of osteoarthritis of the knee, a comparative study between hyaluronic acid and orgatein. E. J. Rheumatol. Inflam., 12, 47-57.

Corrado, E.M., Peluso, G.F., Gigliotti, S., Durante, C.D., Palmieri, D., Savoia, M., Oriani, G.O. \& Tajana, G.F. (1995) The effects of intraarticular administration of hyaluronic acid of the knee. A clinical study with immunological and biochemical evaluation. $E$. J. Rheum. Inflam., 15, 65-75.

Cortivo, R., Brun, P., Cardarelli, L., O'Regan, M., Rdice, M. \& Abatangelo, G. (1996) Antiox- 
idant effects of hyaluronan and its alphamethyl-prednisolone derivative in chondrocyte and cartilage cultures. Semin. Arthritis. Rheum., 26, 492-501.

Frizziero, L., Govoni, E., Zizzi, F., Bacchin, I.P. \& Perbelli, A. (1988) Advances in the arthroscopic analysis of the synovial membrane morphopathology, long-term study of hyaluronic acid treatment in the knee joint osteoarthritis. Scan. J. Immunol., 4, 504-510.

Ghosh, P., Read, R., Armstrong, S., Wilson, D., Marshall, R. \& Mc, N. (1993) The effects of intra-arthicular administration of hyaluronan in a model of early osteoarthritis in sheep I gait analysis and radiological and morphological studies. Semin. Arthritis. Rheum., $\mathbf{5 2}, 817-822$.

Gotoh, S., Onaya, J.I., Abe, M., Miyazaki, K., Hamai, A., Horie, K. \& Tokuyasu, K. (1993) Effects of the molecular weight of hyaluronic acid and its action mechanisms on experimental joint pain in rats. Ann. Rheum. Dis., 52, 817822.

Graf, J., Neusel, E., Schneider, E. \& Niethard, F.U. (1938) Intra-articular treatment with hyaluronic acid in osteoarthritis of the knee joint. Clin. Exp. Rheum., 11, 367-372.

Jones, A.C., Martın, P., Sally, D. \& Michael, D. (1995) Intraarticular hyaluronic acid compared to intra-articular triamcilone hexacetonide in inflammatory knee osteoarthritis. Osteoart. Cart., 8, 269-273.

Karakok, M., Ugras, S., Tosun, N., Akpınar, F. \& Aydin, A. (2001) Effect of intra-articular administration of hyaluronan and cortisone on secondary osteoarthritis of the infected rabbit's knee. Tohoku J. Exp. Med., 195, 35 -42 .

Kvam, B.J., Fragonas, E., Degrassi, A., Kvam, C., Matulova, M., Pollesello, P., Zanetti, F. \& Vittur, F. (1995) Oxygen-derived free radical (ODFR) action on hyaluronan (HA), on two HA ester derivatives, and on the metabolism of articular chondocytes. Exp. Cell Res., 218, 79-86.

Leardini, G., Mattra, L., Franceschini, M. \& Perbelli, A. (1991) Intraarticular treatment of knee osteoarthritis. A comparative study between hyaluronic acid and 6-methylprednisolone acetate. Clin. Exp. Rheum., 9, 375-381.

Marion, G.A. \& Sanford, R.I. (1992) Articular cartilage. In: Histopathology for Pathologisty, edit- ed by S.S. Stenberg, Raven Press, New York, pp. 66-67.

Morales, T.I. \& Hascall, V.C. (1989) Factors involved in the regulation of proteoglycan metabolism in articular cartilage. Arthritis Rheum., 32, 1197-1201.

Namiki, O., Toyoshima, H. \& Morisaki, N. (1982) Therapeutic effect of intraarticular injection of high molecular weight hyaluronic acid on osteoarthritis of the knee. Int. J. Clin. Phar. Ther. Toxicol., 11, 501-507.

Nizolek, D.J.H. \& White, K.K. (1981) Corticosteroid and hyaluronic acid treatment in equine degenerative joint disease a review. Cornell. Vet., 71, 355-375.

Ohkawa, H., Ohishi, N. \& Yagi, K. (1979) Assay for lipid peroxidation in animal tissues by thiobarbituric acid reaction. Anal Biochem., 95, 351-358.

Peyron, J.G. \& Balazs, E.A. (1974) Preliminary clinical assessment of Na-hyaluronate injection into human joints. Path. Biol., 8, 731-736.

Philips, M.W. (1989) Clinical trial comparison of intra-arthicular sodium hyaluronate products in the horse. J. E . Vet. Sci., 9, 39-40.

Pietrogrande, V., Melanotte, P.L., D'agnolo, B., Ulivi, M., Benigni, G.A., Turchetto, L., Pierfederici, P.\& Perbellini, A. (1991) Hyaluronic acid versus methylprednisolone intraarticularly injected for treatment of osteoarthritis of the knee. Current Therapeutic Research, 5, 691701.

Sakakibara, Y., Miura, T., Iwata, H., Kikuchi, T. \& Yamaguchi, T. (1994) Effect of high molecular weight sodium hyaluronate on immobilized rabbit knee. Clin. Orthop. Rel. Res., 299, 282-292.

Selami, Y.D. (1993) Kortizon kullanimi. In: Kortizon Tedavisi, edited by Y.D. Selami, Baskent Press, Ankara, Turkey, pp. 183-192.

Tosun, N., Ugras, S. \& Akpinar, F. (1997) The effect of intraarticular exogenous hyaluronic acid injection on the healing of cartilage damage. Turkish J. Bone Joint Surg., 3, 9698.

Toyoshima, H. (1978) The influence of synoviectomy on articular cartilage of rabbit knee and preventive effects of hyaluronic acid on degenerative changes of the cartilage. $J$. Tokyo Women's Med. Coll., 48, 890-910.

Ustun, M.E., Gurbilek, M., Ak, A., Vatansev, H. \& Duman, A. (2001) Effects of magnesium sul- 
fate on tisuue lactate and malondialdehyde levels in experimental head trauma. Intensive Care Med., 27, 264-268.

Wollheim, F.A. (1999) Serum markers of articular cartilage damage and repair. Rheum. Dis. Clin. North Am., 25, 417-32.

Yoshimi, T.\& Itoh, H. (1994) Effect of highmolecular-weight sodium hyaluronate on immobilized rabit knee. Clin. Orthop. Rel. Res.,
299, 282-292.

Yoshimi, T., Kikuchi, T., Obara, T., Yamaguchi, T., Sakakibara, Y., Itoh, H., Iwata, H. \& Miura, T. (1994) Effects of high molecular weight sodium hyaluronate on experimental osteoarthrosis induced by resection of rabbit anterior crucial ligament. Clin. Orthop. Rel. Res., 298, 296-304. 